\title{
The Empirical Implications of Privacy-Aware Choice*
}

\author{
Rachel Cummings ${ }^{\dagger} \quad$ Federico Echenique $^{\ddagger} \quad$ Adam Wierman $^{\S}$
}

January 3, 2014

\begin{abstract}
This paper initiates the study of the testable implications of choice data in settings where agents have privacy preferences. We adapt the standard conceptualization of consumer choice theory to a situation where the consumer is aware of, and has preferences over, the information revealed by her choices. The main message of the paper is that little can be inferred about consumers' preferences once we introduce the possibility that the consumer has concerns about privacy. This holds even when consumers' privacy preferences are assumed to be monotonic and separable. This motivates the consideration of stronger assumptions and, to that end, we introduce an additive model for privacy preferences that does have testable implications.
\end{abstract}

\section{Introduction}

The purpose of this paper is to study what an observer can learn about a consumer's preferences and behavior when the consumer has concerns for her privacy and knows that she is being observed. The basic message of our results is that very little can be learned without strong assumptions on the form of the consumer's privacy preferences.

To motivate the problem under study, consider the following story. Alice makes choices on the internet. She chooses which websites to visit, what books to buy, which hotels to reserve, and which newspapers to read. She knows, however, that she is being watched. An external agent, "Big Brother" (BB), monitors her choices. BB could be a private firm like Google, or a government agency like the NSA. As a result of being watched, Alice is concerned for her privacy; and this concern affects her behavior.

Alice has definitive preferences over the things she chooses among. For example, given three political blogs $a, b$, and $c$, she may prefer to follow $a$. But, BB will observe such a choice, and infer that she prefers $a$ over $b$ and $c$. This is uncomfortable to Alice, because her preferences are shaped by her political views, and she does not like BB to know her

\footnotetext{
${ }^{*}$ We thank Jeff Ely, and especially Katrina Ligett, for useful comments. This work was supported by NSF grants 1101470, 1216006, and CNS-1254169, as well as BFS grant 2012348 and the Charles Lee Powell Foundation.

${ }^{\dagger}$ Electrical Engineering and Computer Science, Northwestern University. rachelc@u.northwestern.edu

${ }^{\ddagger}$ Humanities and Social Sciences, California Institute of Technology. fede@hss.caltech.edu

${ }^{\S}$ Computing and Mathematical Sciences, California Institute of Technology. adamw@caltech.edu
} 
views or her preferences. As a result, she may be reluctant to choose $a$. She may choose $b$ instead because she is more comfortable with BB believing that she ranks $b$ over $a$ and $c 1$

Now, the question becomes, given observations of Alice's behavior, what can we learn about her preferences? We might conjecture that her behavior must satisfy some kind of rationality axiom, or that one could back out, or reverse-engineer, her preferences from her behavior. After all, Alice is a fully rational consumer (agent), meaning that she maximizes a utility function (or a transitive preference relation). She has a well-defined preference over the objects of choice, meaning that if she could fix what BB learns about her-if what BB learns about her were independent from her choices - then she would choose her favorite object. Further, Alice's preferences over privacy likely satisfy particular structural properties. For example, she has well-defined preferences over the objects of choice, and she cares about the preference revealed by her choices: she always prefers revealing less to revealing more. In economics, preferences of this form are called separable and monotonic; and such preferences normally place strong restrictions on agents' behavior.

However, contrary to the above discussion, the results in this paper prove that nothing can be inferred about Alice's preferences once we introduce the possibility that she has concerns about privacy. No matter what her behavior, it is compatible with some concerns over privacy, i.e., she always has an "alibi" that can explain her choices as a consequence of privacy concerns. The strongest version of this result is that all possible behaviors on the part of Alice are compatible with all possible preferences that Alice may have over objects: postulate some arbitrary behavior for Alice, and some arbitrary preference over objects, and the two will always be compatible.

So BB's objective is hopeless. He can never learn anything about Alice's true preferences over political blogs, or over any other objects of choice. If BB tries to estimate preferences from some given choices by Alice, he finds that all preferences could be used to explain her choices. He cannot narrow down the set of preferences Alice might have, no matter what the observed behavior. The result continues to hold if $\mathrm{BB}$ adversarially sets up scenarios for Alice to choose from. That is, even if BB offers Alice menus of choices so as to maximize what he can learn from her behavior, the result is still that nothing can be learned.

The results in this paper have a variety of implications.

First, they motivate the use of specific parametric models of preferences over privacy. Our main result makes strong qualitative assumptions about preferences (separability, monotonicity). Given that such assumptions lack empirical bite, one should arguably turn to stronger assumptions yet. The paper proposes an additive utility function that depends on the chosen object and on what is revealed by the consumer's choices. If Alice chooses $x$ then she obtains a utility $u(x)$ and a "penalty" $v(x, y)$ for not choosing $y$, for all non-chosen $y$, as she reveals to $\mathrm{BB}$ that she ranks $x$ over $y$. This additive model does have restrictions for the consumer's behavior, and could be estimated given data on Alice's choices. The model is methodologically close to models used in economics to explain individual choices, and could be econometrically estimated using standard techniques.

\footnotetext{
${ }^{1}$ Like Alice, $85 \%$ of adult internet users have take steps to avoid surveillance by other people or organizations, see 8 .
} 
The paper discusses a test for the additive model based on a linear program.

Second, while the paper's main motivation is consumers' behavior on the internet, the results have implications for issues commonly discussed in behavioral economics. Some behavioral "anomalies" could be the consequence of the presence of an outside observer. For example (elaborating on a laboratory experiment by [10]), consider a consumer who is going to buy a technical gadget, such as a phone or a camera. The consumer might prefer a simple camera over a more complex one which they might not know how to operate; but when presented with a menu that has a simple, an intermediate and an advanced camera, they might choose the intermediate one because they do not want to reveal to the world that they do not know how to use a complex camera. Of course, the results show that this line of reasoning may not be very useful, as anything can be explained in this fashion. The results suggest, however, that a stronger parametric model may be useful to explain various behavioral phenomena.

Third, the results explain why BB may want to be hide the fact that consumer behavior is being observed. The NSA or Google seem to dislike openly discussing that they are monitoring consumers' online behavior. One could explain such a desire to hide by political issues, or because the observers wish to maintain a certain public image, but here we point to another reason. The observations simply become ineffective when the consumer is aware that she is being observed.

\section{Modeling privacy preferences}

The goal of this paper is to study the testable implications of choice data in a context where agents have privacy preferences. To this end, we adapt the standard conceptualization of consumer choice theory in economics (see e.g. the textbook treatments in [5] or [9]) to a situation where the consumer is aware of, and has preferences over, the information revealed by her choices.

\subsection{The setting}

We focus on a situation where there is an outside observer (he), such as Google or the NSA, that is gathering data about the choices of a consumer (she) by observing her choices. We assume that the consumer is presented with a set of alternatives $A$ and then makes a choice $c(A)$, which the outside observer sees. The observer then infers from this choice that $c(A)$ is preferred to all other alternatives in $A$.

The above parallels the classical revealed preference theory framework; however our model differs when it comes to the the behavior of the consumer, which we model as "privacy-aware". We assume that the consumer is aware of the existence of an outside observer, and so she may care about what her choices reveal about her. Specifically, her choices are motivated by two considerations. On the one hand, she cares about the actual chosen alternative. On the other hand, she cares about what those choices reveal about her preferences over alternatives, i.e., her revealed preferences. We capture this by assuming that the consumer has preferences over pairs $(x, B)$, where $x$ is the chosen object and $B$ is the information revealed about the consumer's preferences. 
An important point about the setting is that the inferences made by the observer do not recognize that the consumer is privacy aware. This assumption about the observer being naive is literally imposed on the behavior of the observer, but it is really an assumption about how the agent thinks that the observer makes inferences. The agent thinks that the observer naively uses revealed preference theory to make inferences about her preferences. The observer, however, could be as sophisticated as any reader of this paper in how they learn about the agent's preferences. The upshot of our results is that such a sophisticated observer could not learn anything about the agent's behavior.

It is natural to go one step further and ask "What if the agent knows that the observer knows that the agent is privacy-aware?" Or, "what if the agent knows that the observer knows that the agent knows that the observer knows that the agent is privacy-aware?" The problem naturally lends itself to a discussion of the role of higher order beliefs. We formalize exactly this form of a cognitive hierarchy in Section 4 , and we discuss how our results generalize.

\section{$2.2 \quad$ Preliminaries}

Before introducing our model formally, there are a few preliminaries that are important to discuss. Let $\mathbf{B}(X)=2^{X \times X}$ denote the set of all binary preference relations on a set $X$ and recall that a binary relation $\succeq$ is a weak order if it is complete (total) and transitive. We say that $x \succ y$ when $x \succeq y$ and it is not the case that $y \succeq x$. Finally, a linear order is a weak order such that if $x \neq y$ then $x \succ y$ or $y \succ x$.

We shall often interpret binary relations as graphs. For $B \in \mathbf{B}(X)$, define a graph by letting the vertex set of the graph be equal to $X$ and the edge set be $B$. So, for each element $(x, y) \in B$, we have a directed edge in the graph from $x$ to $y$. We say that a binary relation $B$ is acyclic if there does not exist a directed path that both originates and ends at $x$, for any $x \in X$. The following simple result, often called Spilrajn's Lemma, is useful.

Lemma 1. If $B \subseteq \mathbf{B}(X)$ is acyclic, then there is a linear order $\succeq$ such that $B \subseteq \succeq$.

\subsection{The model}

Given the setting described above, our goal is to characterize the testable implications of choice data, and to understand how the testable implications change when consumers are privacy-aware as opposed to privacy-oblivious. To formalize this we denote a choice problem by a tuple $(X, \mathcal{A}, c)$ where $X$ is a finite set of alternatives, $\mathcal{A}$ a collection of nonempty subsets of $X$, and $c: \mathcal{A} \rightarrow X$ such that $c(A) \in A$ for all $A \in \mathcal{A}$.

In choice problem $(X, \mathcal{A}, c)$, the consumer makes choices for each $A \in \mathcal{A}$ according to the function $c$. Further, given $A \in \mathcal{A}$ and $x=c(A)$, the observer infers that the consumer prefers $x$ to any other alternative available in $A$. That is, he infers that the binary comparisons $(x, y) \forall y \in A \backslash\{x\}$ are part of the consumer's preferences over $X$. Such inferences lie at the heart of revealed preference theory (see e.g. [11] or [12]).

A privacy preference is a linear order $\succeq$ over $X \times 2^{X \times X}$. A privacy preference ranks objects of the form $(x, B)$, where $x \in X$ and $B \in \mathbf{B}(X)$. If a consumer's choices are guided 
by a privacy preference, then she cares about two things: she cares about the choice made (i.e. $x$ ) and about what her choices reveal about her preference (hence $B$ ).

Given the notions of a choice problem and privacy preferences defined above, we can now formally define the notion of rationalizability that we consider in this paper.

Definition 1. A choice problem $(X, \mathcal{A}, c)$ is rationalizable (via privacy preferences) if there is a privacy preference $\succeq$ such that if $x=c(A)$ and $y \in A \backslash\{x\}$ then

$$
(x,\{(x, z): z \in A \backslash\{x\}\}) \succ(y,\{(y, z): z \in A \backslash\{y\}\}),
$$

for all $A \in \mathcal{A}$. In this case, we say that $\succeq$ rationalizes $(X, \mathcal{A}, c)$.

Thus, a choice problem is rationalizable when there exists a privacy preference that "explains" the data, i.e., when there exists a privacy preference for which the observed choices are maximal.

\section{The rationalizability of privacy-aware choice}

In this section, we present our main results, which characterize when choice data from privacy-aware consumers is rationalizable. Our results focus on the testable implications of structural assumptions about the form of the privacy preferences of the consumer. While a consumer's preferences may, in general, be a complex combination of preferences over the choices and revealed preferences, there are some natural properties that one may expect to hold in many situations. In particular, we focus on three increasingly strong structural assumptions in the following three subsections: monotonicity, separability, and additivity.

\subsection{Monotone privacy preferences}

A natural assumption on privacy preferences is "monotonicity", i.e., the idea that revealing less information is always better. Monotonicity of privacy preferences is a common assumption in the privacy literature, e.g., see [13] and [6], but of course one can imagine situations where it may not hold, e.g., see [1] and [7].

In our context, we formalize monotone privacy preferences as follows.

Definition 2. A binary relation $\succeq$ over $X \times 2^{X \times X}$ is a monotone privacy preference when

(i) $\succeq$ is a linear order, and

(ii) $B \subsetneq B^{\prime}$ implies that $(x, B) \succ\left(x, B^{\prime}\right)$.

This definition formalizes the idea that revealing less information is better. In particular, if $B \subsetneq B^{\prime}$, then fewer comparisons are being made in $B$ than in $B^{\prime}$, so $(x, B)$ reveals less information to the observer than $\left(x, B^{\prime}\right)$.

Given the above definition, the question we address is "what are the empirical implications of monotone privacy preferences?" That is, "Is monotonicity refutable via choice data?" The following proposition highlights that monotonicity is not refutable, so any choice data has a monotone privacy preference that explains it. 
Proposition 1. Any choice problem is rationalizable via monotone privacy preferences.

Proof. We shall use the following notation:

$$
A_{x}=\{(x, y): y \in A \backslash\{x\}\}
$$

Define a binary relation $E$ on $X \times \mathbf{B}(X)$ as follows: $(x, B) E\left(x^{\prime}, B^{\prime}\right)$ if either $x=x^{\prime}$ and $B \subsetneq B^{\prime}$, or $x \neq x^{\prime}$ and there is $A \in \mathcal{A}$ with $x=c(A), x^{\prime} \in A$ and $B=A_{X}$ while $B^{\prime}=A_{x^{\prime}}$. It will be useful for our proof to think of $E$ as the edges of a directed graph $G=(V, E)$, where $V=X \times \mathbf{B}(X)$. The edges where $x=x^{\prime}$ result from the monotonicity requirement, and the edges where $x \neq x^{\prime}$ result from the requirement that observed choices be rationalized. For shorthand, we will call these edges "monotone" and "rationalizing," respectively. It should be clear that any linear order that extends $B$ (i.e any linear order $\succeq$ with $E \subseteq \succeq)$ is a monotone privacy preference that rationalizes $(X, \mathcal{A}, c)$. By Lemma 11, we are done if we show that $E$ is acyclic. To prove that $E$ is acyclic, it is equivalent to show that the graph is acyclic.

By the definition of $E$, for any pair $(x, B) E\left(x^{\prime}, B^{\prime}\right)$, the cardinality of $B$ must be at most that of $B^{\prime}$, and if $x=x^{\prime}$ then the cardinality must be strictly smaller due to monotonicity. Hence, there can be no cycles containing monotone edges.

Thus any cycle must contain only rationalizing edges $(x, B) E\left(x^{\prime}, B^{\prime}\right)$ with $x \neq x^{\prime}$. Each such edge arises from some $A \in \mathcal{A}$ for which $B=A_{X}$ while $B^{\prime}=A_{x^{\prime}}$, and for each such $A$ there is a unique $x \in A$ with $x=c(A)$. If the graph were to contain two consecutive rationalizing edges, it would contradict uniqueness of choice. Therefore there cannot be any cycles in $E$.

Proposition 1 provides a contrast to the context of classical revealed preference theory, when consumers are privacy-oblivious. In particular, in the classical setting, choice behavior that violates the strong axiom of revealed preferences (SARP) is not rationalizable, and thus refutes the consumer choice model. However, when privacy-aware consumers are considered, such a refutation of monotonic preferences is impossible. Interestingly, this means that while one may believe that preferences are non-monotonic, the form of data considered in this paper does not have the power to refute monotonicity 2

Note that the question addressed by Proposition 1 is only whether the consumer's choice behavior is consistent with rational behavior, and is not about whether the consumer's underlying preferences over outcomes in $X$ can be learned. In particular, these underlying preferences may not even be well defined for the general model considered to this point. We address this issue in the next section after imposing more structure on the privacy preferences.

\subsection{Separable privacy preferences}

That all choice behavior is rationalizable via monotone privacy preferences can be attributed to the flexibility provided by such preferences. Here we turn to a significant restriction on the preferences one might use in rationalizing the consumer's behavior.

\footnotetext{
${ }^{2}$ This phenomenon is common in the consumer choice formulation of the revealed preference problem, but it comes about for completely different reasons.
} 
It is natural to postulate that the consumer would have some underlying, or intrinsic, preferences over possible options when her choices are not observed. Indeed, the observer is presumably trying to learn the agent's preferences over objects. Such preferences should be well defined: if outcome $x$ is preferred to outcome $y$ when both are paired with the same privacy set $B$, then it is natural that $x$ will always be preferred to $y$ when both are paired with the same privacy set $B^{\prime}$, for all possible $B^{\prime}$. This property induces underlying preferences over items in $X$, as well as the agent's privacy-aware preferences.

We formalize the notion of separable privacy preferences as follows.

Definition 3. A binary relation $\succeq$ over $X \times 2^{X \times X}$ is a separable privacy preference if it is a monotone privacy preference and additionally satisfies that for all $x, y \in X$ and $B \in \mathbf{B}(X)$,

$$
(x, B) \succeq(y, B) \Longrightarrow\left(x, B^{\prime}\right) \succeq\left(y, B^{\prime}\right) \forall B^{\prime} \in \mathbf{B}(X)
$$

That is, whenever $(x, B)$ is preferred to $(y, B)$ for some preference set $B$, then also $\left(x, B^{\prime}\right)$ is preferred to $\left(y, B^{\prime}\right)$ for all other sets $B^{\prime}$.

Separable privacy preferences have an associated preference relation over $X$. If $\succeq$ is a separable privacy preference, then define $\left.\succeq\right|_{X}$ as $\left.x \succeq\right|_{X} y$ if and only if $(x, B) \succsim(y, B)$ for all $B \in \mathbf{B}(X)$. Note that $\left.\succeq\right|_{X}$ is a linear order over $X$. We can interpret $\left.\succeq\right|_{X}$ as the projection of $\succeq$ onto $X$.

There are two questions we seek to answer: "What are the empirical implications of separability?" and "When can an observer learn the underlying choice preferences of the consumer?" The following proposition addresses both of these questions. Note that Proposition 2 follows from a more general result, Theorem 1, which is presented in Section 4.

Proposition 2. Let $(X, \mathcal{A}, c)$ be a choice problem, and let $\succeq$ be any linear order over $X$. Then there is a separable privacy preference $\succeq^{*}$ that rationalizes $(X, \mathcal{A}, c)$ such that the projection of $\succeq^{*}$ onto $X$ is well defined and coincides with $\succeq$, i.e., $\left.\succeq^{*}\right|_{X}=\succeq$.

Think of $\succeq$ as a conjecture that the observer has about the agent. Proposition 2 implies that no matter the nature of such a conjecture, and no matter what choice behavior is observed, the two are compatible.

This proposition carries considerably more weight than Proposition 1. Separability imposes much more structure than monotonicity alone and, further, Proposition 2 says much more than simply that separability has no testable implications, or that it is not refutable via choice data. Proposition 2 highlights that the task of the observer is hopeless in this case - regardless of the choice data, there are preferences over revealed information that allow all possible choice observations to be explained.

That is, the choice data does not allow the observer to narrow his hypothesis about the consumer preferences at all. This is because the consumer always has an alibi available (in the form of preferences over revealed information) which can allow her to make the observed data consistent with any preference ordering over choices.

In some sense, our result is consistent with the idea that secrecy is crucial for observers such as the NSA and Google. If the consumer is not aware of the fact that she is being observed then the observer can learn a considerable amount from choice data, while if the 
consumer is aware that she is being observed then the choice data has little power (unless more structure is assumed than separability).

\subsection{Additive privacy preferences}

So far, we have seen that monotonicity and separability do not provide enough structure to allow choice data to have testable implications or to allow the observer to learn anything about consumer preferences over choices. This implies that further structure must be imposed for choice data to have empirical power. To that end, we now give an example of a model for privacy preferences where choice data does have testable implications. The model we consider builds on the notion of separable privacy preferences and additionally imposes additivity.

Definition 4. A binary relation $\succeq$ over $X \times 2^{X \times X}$ is an additive privacy preference if there are functions $u: X \rightarrow \mathbb{R}^{+}$and $v: X \times X \rightarrow \mathbb{R}^{+}$such that $(x, B) \succ\left(x^{\prime}, B^{\prime}\right)$ iff

$$
u(x)-\sum_{\left(z, z^{\prime}\right) \in B} v\left(z, z^{\prime}\right)>u\left(x^{\prime}\right)-\sum_{\left(z, z^{\prime}\right) \in B^{\prime}} v\left(z, z^{\prime}\right) .
$$

While monotonicity and separability are general structural properties of privacy preferences, the definition of additivity is much more concrete. It specifies a particular functional form, albeit a simple and natural one. In this definition, the consumer experiences utility $u(x)$ from the choice made and disutility $v(x, y)$ from the privacy loss of revealing that $x \succ y$ for every pair $(x, y) \in X \times X$. Note that this form is an additive extension of the classical consumer choice model, which would include only $u$ and not $v$.

Moreover, this definition also satisfies both monotonicity and separability, making it a strictly stronger restriction. Monotonicity is satisfied because the agent always experiences a loss from each preference inferred by the observer. Namely, the range of $v$ is restricted to non-negative reals, so for a fixed choice element, the agent will always prefer fewer inferences to be made about her preferences 3 Separability is satisfied because utilities $u$ determine the linear ordering over $X$, so for a fixed set of inferences made by the observer, privacy preferences will correspond to the preferences determined by $u$.

Of course there are a number of variations of this form that could also make sense, e.g., if the disutility from a revealed preference $(x, y)$ was only counted once instead of (possibly) multiple times due to multiple revelations in the choice data. This would correspond to a consumer maximizing a "global" privacy loss rather than optimizing online for each menu. However, this modeling choice requires the agent to know ex ante the set $\mathcal{A}$ of menus from which she will choose, and additional assumptions about the order in which the she faces these menus. For our analysis we restrict to additive preferences as defined above.

Rationalizability of additive privacy preferences corresponds to the existence of functions $u$ and $v$, such that the observed choice behavior maximizes the consumer's utility under these functions. Here, it turns out the imposed structure on privacy preferences

\footnotetext{
${ }^{3}$ Monotonicity restricts to the case where people want to keep their preferences private. It may be interesting to explore in future work, the case where people are happy to reveal their information, e.g., conspicuous consumption. Under additive preferences, this would correspond to allowing the range of $v$ to be all of $\mathbb{R}$.
} 
is enough to allow the model to have testable implications, as shown in the following proposition.

Proposition 3. There exists a choice problem $(X, \mathcal{A}, c)$ that is not rationalizable with additive privacy preferences.

Proposition 3 highlights that, while monotonicity and separability cannot be refuted with choice data, additivity can be refuted. To show this, we construct a simple example of choice data that cannot be explained with any functions $u$ and $v$.

Proof of Proposition 3. To construct an example that is not rationalizable via additive privacy preferences, we begin by defining the set of alternatives as $X=\{x, y, z, w\}$ and the choice data as follows. It includes six observations: $z=c(\{x, z\}), x=c(\{x, y, z\})$, $w=c(\{w, z\}), z=c(\{w, y, z\}), x=c(\{x, w\}), w=c(\{x, y, w\})$.

To see that this choice data is not rationalizable suppose, towards a contradiction, that the pair $(u, v)$ rationalizes $c$. Then $z=c(\{x, z\})$ implies that

$$
u(z)-v(z, x)>u(x)-v(x, z),
$$

while $x=c(\{x, y, z\})$ implies that

$$
u(z)-v(z, x)-v(z, y)<u(x)-v(x, z)-v(x, y) .
$$

Therefore $v(z, y)>v(x, y)$.

Similarly, we can argue that $w=c(\{w, z\})$ and $z=c(\{w, y, z\})$ together imply that $v(w, y)>v(z, y)$, and $x=c(\{x, w\})$ and $w=c(\{x, y, w\})$ together imply that $v(x, y)>v(w, y)$. This gives us a contradiction and so proves that the choice data is not rationalizable.

Given that the structure imposed by additive privacy preferences is testable, the next task is to characterize data sets that are consistent with (or refute) the additive privacy preference model. The example given in the proof of Proposition 3 already suggests an important feature of choice data that must hold for it to be rationalizable.

Given a choice problem $(X, \mathcal{A}, c)$ and an element $y \in X$, define the binary relation $R^{y}$ by $x R^{y} z$ if there is $A \in \mathcal{A}$ with $z=c(A)$ and $x=c(A \cup\{y\})$. Our next result gives a test for additively rational preferences. It says that, if there are cycles in the binary relation $R^{y}$, then the choice data cannot be rationalized by additive privacy preferences.

Proposition 4. A choice problem can be rationalized by additive privacy preferences only if $R^{y}$ is acyclic, for all $y$.

Proof. Let $c$ be rationalizable by the additive privacy preferences characterized by $(u, v)$. For each $x, z \in X$ such that $x R^{y} z$, then there is some $A \in \mathcal{A}$ such that $z=c(A)$ and $x \in A$, so

$$
u(z)-\sum_{t \in A} v(z, t)>u(x)-\sum_{t \in A} v(x, t)
$$


Similarly, $x=c(A \cup\{y\})$ and $z \in A \cup\{y\}$, so

$$
u(z)-\sum_{t \in A} v(z, t)-v(z, y)>u(x)-\sum_{t \in A} v(x, t)-v(x, y) .
$$

For both inequalities to be true simultaneously, we need $v(z, y)>v(x, y)$. Thus,

$$
x R^{y} z \Longrightarrow v(z, y)>v(x, y) \text {. }
$$

Now assume there exists a cycle in binary relation $R^{y}: a_{1} R^{y} a_{2} R^{y} \cdots R^{y} a_{k} R^{y} a_{1}$. Then by Equation (10), it must be that $v\left(a_{1}, y\right)>v\left(a_{2}, y\right)>\cdots>v\left(a_{k}, y\right)>v\left(a_{1}, y\right)$. In particular, $v\left(a_{1}, y\right)>v\left(a_{1}, y\right)$ which is a contradiction. Then for choices to be rationalized, acyclicity of $R^{y}$ for all $y \in X$ is a necessary condition.

Of course, one would like to develop a test for rationalizability that is both necessary and sufficient. We do this next. Unfortunately, the test we develop takes super-exponential time to even write down. This suggests that acyclicity of $R^{y}$, despite being only a necessary condition, is likely a more practical condition to use when testing for rationalizability.

To describe the test for rationalizability, first observe that when an object $x$ is chosen from a set, the observer infers that $x$ (with its associated privacy) is preferred to $y$ (and its associated privacy), for all $y \in A \backslash\{x\}$. Since we have assumed these preferences to have a specific functional form as in Definition 4, the observer can also infer the corresponding inequality in terms of functions $u$ and $v$. We initialize a large matrix to record the inequalities that are inferred from choice behavior, and ask if there exist values of $u(x)$ and $v\left(x, x^{\prime}\right)$ for all $x, x^{\prime} \in X$ for which all inferred inequalities hold. If so, these values of $u$ and $v$ form additive privacy preferences that rationalize choices. If not, then no such preferences exist and the observed choice behavior is not rationalizable.

Proposition 5. A choice problem $(X, \mathcal{A}, c)$ is rationalizable if and only if there exists functions $u: X \rightarrow \mathbb{R}^{+}$and $v: X \times X \rightarrow \mathbb{R}^{+}$satisfying the matrix inequality given by equation (3).

Proof. For ease of notation, index the elements of $X=\left\{x_{1}, \ldots, x_{n}\right\}$. Then for each $A \in \mathcal{A}$, the agent chooses some $x_{i}=c(A) \in A$. By the definition of additive preferences, every $x_{j} \in A$ for $j \neq i$ was not chosen because

$$
u\left(x_{i}\right)-\sum_{z \in A \backslash\left\{x_{i}\right\}} v\left(x_{i}, z\right)>u\left(x_{j}\right)-\sum_{z \in A \backslash\left\{x_{j}\right\}} v\left(x_{j}, z\right)
$$

Rearranging terms gives,

$$
u\left(x_{i}\right)-u\left(x_{j}\right)+\sum_{z \in A \backslash\left\{x_{j}\right\}} v\left(x_{j}, z\right)-\sum_{z \in A \backslash\left\{x_{i}\right\}} v\left(x_{i}, z\right)>0
$$

We can instantiate a matrix $T$ to record the inequalities implied by all observed choices. This matrix $T$ will have $n^{2}$ columns, where the first $n$ columns correspond to elements $x_{1}, \ldots, x_{n} \in X$, and the remaining $n^{2}-n$ columns correspond to ordered pairs $\left(x_{i}, x_{j}\right)$ of 
elements in $X$, for $i \neq j 4 T$ will contain a row for each triple $\left(A, x_{i}, x_{j}\right)$, where $A \in \mathcal{A}$, and $x_{i}, x_{j} \in A$. If the agent is observed to choose $x_{i}=c(A)$, then Equation (2) must be true for each $x_{j} \in A$ for $j \neq i$. To encode this inequality, then for each such $x_{j}$ that was not chosen from $A$, we fill in the row corresponding to $\left(A, x_{i}, x_{j}\right)$ as follows: enter +1 in the $i^{\text {th }}$ column, -1 in the $j^{\text {th }}$ column, +1 in columns corresponding to pairs $\left(x_{j}, z\right)$ where $z \in A,-1$ in columns corresponding to pairs $\left(x_{i}, z\right)$ where $z \in A$, and zeros elsewhere.

To complete the encoding, we also require a vector $\vec{u}$, which contains the values of $u(\cdot)$ and $v(\cdot, \cdot)$ evaluated on all elements of $X$. The first $n$ entries of $\vec{u}$ will contain the values of $u\left(x_{1}\right), \ldots, u\left(x_{n}\right)$, and the remaining $n^{2}-n$ entries will contain the values $v\left(x_{i}, x_{j}\right)$ for $i \neq j$, in the same order in which the pairs appear in the columns of $T$. Then multiplying $T \vec{u}$, each row of the product would equal

$$
u\left(x_{i}\right)-u\left(x_{j}\right)+\sum_{z \in A \backslash\left\{x_{j}\right\}} v\left(x_{j}, z\right)-\sum_{z \in A \backslash\left\{x_{i}\right\}} v\left(x_{i}, z\right)
$$

for some set $A \in \mathcal{A}$, observed choice $x_{i}=c(A)$, and unchosen element $x_{j} \in A$. Finally, we need the constraint that each row of $T \vec{u}$ is greater than zero, as required in Equation 2 , That is,

$$
T \vec{u}>\overrightarrow{0}
$$

If such a vector $\vec{u}$ exists, then there exist functions $u: X \rightarrow \mathbb{R}^{+}$and $v: X \times X \rightarrow \mathbb{R}^{+}$ such that additive privacy preferences are optimized by the observed choices, and the thus observed choices are rationalizable.

\section{$4 \quad$ Higher order privacy preferences}

The results we have discussed so far are predicated on the notion that the agent thinks that the observer is naive. We shall now relax the assumption of naivete. We are going to allow the agent to believe that the observer thinks that she is privacy aware.

Going back to Alice, who is choosing among political blogs, suppose that she reasons as follows. Alice may realize that her observed choices violate the strong axiom of revealed preference and therefore cannot correspond to the choices of a rational agent. This could tip off the observer to the fact that she is privacy aware. We have seen that privacy awareness is a plausible explanation for violations of the revealed preference axioms. So Alice could now be concerned about the observer's inference about her preferences over objects and over revealed preference. Perhaps she thinks that the observer will infer that she is avoiding blog $a$ because of what it reveals about her, and that fact itself is something she does not wish be known. After all, if Alice has a preference for privacy, perhaps she has something to hide.

More generally, an agent may be concerned not only about what her behavior reveals about her preferences over $X$, but also about what her behavior reveals of her preferences for privacy. She may then make choices to minimize inferences the observer is able to make about her preferences for privacy, as well as her preferences over $X$.

\footnotetext{
${ }^{4}$ There are only $n^{2}-n$ columns because we do not compare elements to themselves
} 
To provide a model that incorporates such issues, we define a hierarchy of higher order preferences, called level- $k$ preferences, where a level- $k$ consumer is aware that the observer may make inferences about her level- $(k-1)$ privacy preferences, and has preferences over the information the observer can infer. In our construction, level-0 corresponds to the classical privacy-oblivious setting, and the setting we have considered to this point is that of a level-1 consumer (Sections 2 and 3).

The meaning of such levels should be clear. If Alice is concerned about facing an observer who makes level $k$ inferences, then her behavior will be dictated by the level $k+1$ model. To emphasize a point we have made repeatedly, the real observer may be as sophisticated as one wants, but Alice thinks that the observer thinks that Alice thinks that the observer thinks that Alice thinks ... that the observer makes inferences based on revealed preferences.

\subsection{Level- $k$ privacy preferences}

To formally define a "cognitive hierarchy" for privacy-aware consumers we use the following sequence of sets, $\mathcal{Y}^{k}$ for $k \geq 0$. $\mathcal{Y}^{0}=X, \mathcal{Y}^{1}=X \times \mathbf{B}\left(\mathcal{Y}^{0}\right)$, and let $\mathcal{Y}^{k}=X \times \mathbf{B}\left(\mathcal{Y}^{k-1}\right)$. A level- $k$ privacy preference can then be defined as a binary relation $\succeq^{k}$ over $\mathcal{Y}^{k}=X \times$ $\mathbf{B}\left(\mathcal{Y}^{k-1}\right)$. That is, $\succeq^{k}$ describes preferences over pairs of objects $x \in X$ and the set of level- $(k-1)$ preferences that are revealed from the choice of $x$.

Given the results in Section 3, our focus is on monotone, separable privacy preferences, and so we can extend the notion of monotonicity discussed in Section 3 to level- $k$ privacy preferences as follows.

Definition 5. A monotone level- $k$ privacy preference is a binary relation $\succeq^{k}$ over $\mathcal{Y}^{k}=X \times \mathbf{B}\left(\mathcal{Y}^{k-1}\right)$ such that

1. $\succeq^{k}$ is a linear order, and

2. $B \subsetneq B^{\prime}$ implies that $(x, B) \succ\left(x, B^{\prime}\right)$, for all $B, B^{\prime} \in \mathbf{B}\left(\mathcal{Y}^{k-1}\right)$.

For this definition to hold for level-0, we define $\mathcal{Y}^{-1}$ to be the empty set.

Similarly, we extend the notion of separability to level- $k$ privacy preferences as follows.

Definition 6. A separable level- $k$ privacy preference is a binary relation $\succeq^{k}$ over $\mathcal{Y}^{k}=X \times \mathbf{B}\left(\mathcal{Y}^{k-1}\right)$ such that it is monotone and additionally satisfies for any $B \in$ $\mathbf{B}\left(\mathcal{Y}^{k-1}\right)$,

$$
(x, B) \succeq^{k}(y, B) \Longrightarrow\left(x, B^{\prime}\right) \succeq^{k}\left(y, B^{\prime}\right) \forall B^{\prime} \in \mathbf{B}\left(\mathcal{Y}^{k-1}\right)
$$

Given the notion of level- $k$ privacy preferences, we need to characterize how an observer will make inferences from observed choices. Naturally, the exact information inferred will depend on the level which the observer believes the privacy preferences to be. For example, if the observer believes the consumer to have level-0 preferences, the information inferred by the observer is the set

$$
A_{x}=\{(x, y): y \in A \backslash\{x\}\},
$$


which is a binary relation over $X$. So $A_{x} \in \mathbf{B}\left(\mathcal{Y}^{0}\right)$. However, if the observer believes the consumer to have level-1 preferences, the information inferred by the observer is the set

$$
\left\{\left(\left(x, A_{x}\right),\left(y, A_{y}\right)\right): y \in A \backslash\{x\}\right\} \in \mathbf{B}\left(\mathcal{Y}^{1}\right) .
$$

More generally, to describe the observer's inferences under the the belief that the consumer is level- $k$, we introduce the following notation. Consider the functions $T^{k}$ : $\mathcal{A} \times X \rightarrow \mathbf{B}\left(\mathcal{Y}^{k}\right)$, for $k \geq 0$. Let

$$
\begin{aligned}
T^{0}(A, x)= & \{(x, y): y \in A \backslash\{x\}\} \in \mathbf{B}\left(\mathcal{Y}^{0}\right) \\
T^{1}(A, x)= & \left\{\left(\left(x, T^{0}(A, x)\right),\left(y, T^{0}(A, y)\right)\right): y \in A \backslash\{x\}\right\} \in \mathbf{B}\left(\mathcal{Y}^{1}\right) \\
\vdots & \vdots \\
T^{k}(A, x)= & \left\{\left(\left(x, T^{k-1}(A, x)\right),\left(y, T^{k-1}(A, y)\right)\right): y \in A \backslash\{x\}\right\} \in \mathbf{B}\left(\mathcal{Y}^{k}\right) .
\end{aligned}
$$

In words, $T^{k}(A, x)$ are the level- $k$ preferences (over alternatives in $A$ and set of level$(k-1)$ preferences that will be inferred from each choice) that would cause the agent to choose $x$ from the set $A$. Then generally, a level- $k$ agent making choice $x=c(A)$ must have $T^{k}(A, x)$ as a subset of her level- $k$ preferences.

\section{Example: Level-2 privacy preferences}

In order to illustrate the cognitive hierarchy more concretely it is useful to describe the case of level-2 privacy preferences in detail. Recall that the level-0 privacy preferences are the classical setting of privacy-oblivious consumers and level-1 privacy preferences are the case we study in Sections 2 and 3. As we shall see, there is a sense in which level-2 is all that is needed.

Continuing with the story about Alice, we remarked how she could come to question her level-1 behavior because she should realize that there is something suspicious about her choices violating the revealed preference axioms. As the result of such a realization, she might entertain level-2 behavior. She might think that the observer thinks that she is level-1. Now, there is no reason for her to go any further because, in contrast with level-1, nothing could give her away.

While her violations of the revealed preference axioms indicate that she cannot be level0 , given our Proposition 1, nothing about her behavior could contradict that she is level-1. She has no reason to think that reasoning beyond level-2 will afford her more privacy - we have already seen that nothing in her behavior that could prove to the observer that she is not level-1.

More concretely, suppose that $x$ is chosen from set $A$. The observer, who thinks the consumer is at level-1, infers the level-1 preferences

$$
\left(x, A_{x}\right) \succ\left(z, A_{z}\right) \forall z \in A \backslash\{x\},
$$

or, more specifically, that her level-1 privacy preferences correspond to the binary relation,

$$
\bigcup\left\{\left[\left(x, A_{x}\right),\left(z, A_{z}\right)\right]: z \in A \backslash\{x\}\right\} \text {. }
$$


Now the agent who believes that the observer will make such an inference, will only choose $x$ when this choice together with inferences revealed by the choice is better than the choice of another alternative in $A$ with its accompanying inferences. That is, she will choose $x$ over $y$ in $A$ whenever the choices of $x$ together with the release of the information in Equation (4) is preferred to the choice of $y$ together with the information,

$$
\bigcup\left\{\left[\left(y, A_{y}\right),\left(z, A_{z}\right)\right]: z \in A \backslash\{y\}\right\} .
$$

That is, if a level 2 agent chooses $x$ from set $A$, she knows that observer will make inferences according to Equation (44). Then her choice of $x$ must maximize her preferences over outcomes and these known inferences that will be made. Specifically, she will choose $x$ if her level-2 preferences are, for all available $y \in A$,

$$
\left(x, \cup\left\{\left[\left(x, A_{x}\right),\left(z, A_{z}\right)\right]: z \in A \backslash\{x\}\right\}\right) \succ\left(y, \cup\left\{\left[\left(y, A_{y}\right),\left(z, A_{z}\right)\right]: z \in A \backslash\{z\}\right\}\right)
$$

Using the notation defined earlier in this section, we can re-write Equation (5) as a binary relation,

$$
\left[\left(x, T^{1}(A, x)\right),\left(y, T^{1}(A, y)\right)\right] .
$$

Since the same can be said for every available alternative $y \in A$ that was not chosen, the following must be a part of the agent's level-2 preferences

$$
T^{2}(A, x)=\left\{\left[\left(x, T^{1}(A, x)\right),\left(y, T^{1}(A, y)\right)\right]: y \in A \backslash\{x\}\right\}
$$

Note, however, that the observer does not get to infer $T^{2}(A, x)$. He believes the agent to have level-1 preferences, and upon seeing $x=c(A)$, he infers $T^{1}(A, x)$. This is why the agent chooses $x \in A$ to optimize her preferences over $X$ and sets of the form $T^{1}(A, \cdot)$.

\subsection{The rationalizability of level- $k$ preferences}

Given the notion of a privacy-aware cognitive hierarchy formalized by level- $k$ privacy preferences, we are now ready to move on to the task of understanding the empirical implications of higher order reasoning by privacy-aware consumers. To do this, we must first adapt the notion of rationalizability to level- $k$ reasoning. For this, the natural generalization of Definition 1 to higher order reasoning is as follows. This definition reduces to Definition 1 when level-1 is considered, and to the classical definition of rationalizable in the privacy-oblivious case when level-0 is considered.

Definition 7. A choice $(X, \mathcal{A}, c)$ is level- $k$ rationalizable if there is a level- $k$ privacy preference $\succeq^{k} \in \mathbf{B}\left(\mathcal{Y}^{k}\right)$ such that for all $A \in \mathcal{A}, T^{k}(A, c(A)) \subseteq \succeq^{k}$.

Given this definition, we can now ask the same two questions we considered in Section 3 about level- $k$ privacy preferences: "What are the empirical implications of level- $k$ privacy preferences?" and "When can the observer learn the underlying choice preferences of consumers?" Our main result is the following theorem, which answer these questions. 
Theorem 1. Let $(X, \mathcal{A}, c)$ be a choice problem. Let $k>0$ and $\succeq$ be any linear order over $X$. Then there is a monotone, separable level-k privacy preference $\succeq^{*}$ that level- $k$ rationalizes $(X, \mathcal{A}, c)$ and such that:

$$
x \succeq y \text { iff }(x, B) \succeq^{*}(y, B) \text { for all } B \in \mathbf{B}\left(\mathcal{Y}^{k-1}\right) .
$$

Proof. Let $T^{k-1}: \mathcal{A} \times X \rightarrow \mathbf{B}\left(\mathcal{Y}^{k-1}\right)$ be as defined in Section 4.1, For shorthand, write $\mathcal{Y}$ for $\mathcal{Y}^{k-1}$ and $T$ for $T^{k-1}$. Then $T$ describes the set of level- $(k-1)$ preferences inferred by the observer as a result of the agent's choice behavior. That is, when the agent chooses $x=c(A)$, the observer will infer all preferences in the set $T(A, x)$. Note that $T$ is one-toone and satisfies the following property: for all $A \in \mathcal{A}$ and all $x, x^{\prime} \in A$,

$$
|T(A, x)|=\left|T\left(A, x^{\prime}\right)\right|
$$

Property (6) follows because the number of pairs $((x, T(A, x)),(y, T(A, y))) y \in A \backslash\{x\}\}$ is the same for any $x \in A$.

We now construct a binary relation $E$ over $X \times \mathbf{B}(\mathcal{Y})$. As in the proof of Proposition 1, it will be useful to consider $E$ as the edges of a directed graph $G=(V, E)$, where $V=X \times$ $\mathbf{B}(\mathcal{Y})$. We create edges in $E$ according to the desiderata of our privacy-aware preferences: monotone, separable, and rationalizing choice behavior. Define $E$ as follows: $(x, B) E$ $\left(x^{\prime}, B^{\prime}\right)$ if either (1) $x=x^{\prime}$ and $B \subsetneq B^{\prime},(2)(x, B) E\left(x^{\prime}, B\right)$, where $x \succ x^{\prime}$ according to linear order $\succeq$, or (3) $x \neq x^{\prime}$ and there is $A \in \mathcal{A}$ with $x=c(A), x^{\prime} \in A$ and $B=T(A, x)$ while $B^{\prime}=T\left(A, x^{\prime}\right)$. We will call these edges respectively "monotone," "separable," and "rationalizing," as a reference to the property they are meant to impose. By Lemma 1, we are done if we show that $E$ is acyclic.

Assume towards a contradiction that there is a cycle in this graph. Then there exists a sequence $j=1, \ldots, K$ such that

$$
\left(x^{1}, B^{1}\right) E\left(x^{2}, B^{2}\right) E \cdots E\left(x^{K}, B^{K}\right) \text { and }\left(x^{K}, B^{K}\right) E\left(x^{1}, B^{1}\right) .
$$

For any monotone edge $\left(x^{i}, B^{i}\right) E\left(x^{i+1}, B^{i+1}\right)$, it must be the case that $\left|B^{i}\right|<\left|B^{i+1}\right|$ since $B^{i} \subset B^{i+1}$. If this were a separable edge, then $B^{i}=B^{i+1}$, so $\left|B^{i}\right|=\left|B^{i+1}\right|$. Similarly, for any rationalizing edge, $\left|B^{i}\right|=|T(A, x)|=\left|T\left(A, x^{\prime}\right)\right|=\left|B^{i+1}\right|$. Thus as we traverse any path in this graph, the size of the second component is non-increasing along all edges, and strictly decreasing along monotone edges. This implies that there can be no cycles containing monotone edges, and our assumed cycle must consist entirely of rationalizing and separable edges.

If there are two sequential rationalizing edges in this cycle, then there exists a $j$ and some $A \in \mathcal{A}$ such that

$$
\left(x^{j}, T\left(A, x^{j}\right)\right) E\left(x^{j+1}, T\left(A, x^{j+1}\right)\right) E\left(x^{j+2}, T\left(A, x^{j+2}\right)\right),
$$

where $x^{j}, x^{j+1}, x^{j+2} \in A$. From the first edge, $x^{j}=c(A)$ in some observation, and from the second edge, $x^{j+1}=c(A)$ in another observation. If $x^{j} \neq x^{j+1}$ then $c(A)=x^{j} \neq$ $x^{j+1}=c(A)$ which contradicts the uniqueness of choice imposed by the linear ordering. If $x^{j}=x^{j+1}$, then $\left(x^{j}, T\left(A, x^{j}\right)\right)=\left(x^{j+1}, T\left(A, x^{j+1}\right)\right)$, which implies that an element of 
$X \times \mathbf{B}(\mathcal{Y})$ is strictly preferred to itself, which is a contradiction. Thus no cycle can contain two sequential rationalizing edges.

If there are two sequential separable edges in our cycle, then there exists a $j$ such that

$$
\left(x^{j}, B^{j}\right) E\left(x^{j+1}, B^{j+1}\right) E\left(x^{j+2}, B^{j+2}\right),
$$

where $B^{j}=B^{j+1}=B^{j+2}$ and $x^{j} \succ x^{j+1} \succ x^{j+2}$. By transitivity, $x^{j} \succ x^{j+2}$, so there must also be a separable edge in the graph $\left(x^{j}, B^{j}\right) E\left(x^{j+2}, B^{j+2}\right)$. If the cycle we have selected contains two sequential separable edges, then there must exist another cycle that is identical to the original cycle, except with the sequential separable edges replaced by the single separable edge. Thus we can assume without loss of generality that the cycle we have selected does not contain two sequential separable edges 5

Given the previous two observations, we can assume without loss of generality that the cycle $j=1, \ldots, K$ contains alternating rationalizing and separable edges. This includes the endpoints $j=K$ and $j=1$ since they too are connected by an edge.

If there is a path in the graph containing sequential rationalizing, separable, and rationalizing edges, then there exists $A, A^{\prime} \in \mathcal{A}$ and a $j$ such that

$$
\left(x^{j}, T\left(A, x^{j}\right)\right) E\left(x^{j+1}, T\left(A, x^{j+1}\right)\right) E\left(x^{j+2}, T\left(A^{\prime}, x^{j+2}\right)\right) E\left(x^{j+3}, T\left(A^{\prime}, x^{j+3}\right)\right),
$$

where $x^{j}, x^{j+1} \in A, x^{j+2}, x^{j+3} \in A^{\prime}$, and choices $x^{j}=c(A)$ and $x^{j+2}=c\left(A^{\prime}\right)$ are observed. Since the middle edge is separable, it must be that $T\left(A, x^{j+1}\right)=T\left(A^{\prime}, x^{j+2}\right)$, so $x^{j+1}=$ $x^{j+2}$ and $A=A^{\prime}$. However, this means that $\left(x^{j+1}, T\left(A, x^{j+1}\right)\right)$ is strictly preferred to itself, which is a contradiction, so no such path in the graph can exist.

Since edges must alternate between rationalizing and separable, this leaves the only possible cycles to be of length two, containing one rationalizing edge and one separable edge. However, if such a cycle existed, then traversing the cycle twice would yield a path containing sequential rationalizing, separable, and rationalizing edges, which has been shown to not exist in this graph.

Thus we can conclude that $E$ must be acyclic, which completes the proof.

\section{Related work}

The growing attention to privacy concerns has led to a growing literature studying privacy, see [4] for a survey. Within this literature, an important question is how to model the preferences or utilities of privacy-aware agents in a way that describes their behavior in strategic settings.

One approach toward this goal, exemplified by [2, 6], 1], [13], and [7, is to use differential privacy in mechanism design as a way to quantify the privacy loss of an agent from participating the mechanism. Within this literature, each of [2], [6], and [13] assume that the utility of a privacy-aware agent is her gain from the outcome of the interaction minus her loss from privacy leakage. Note that this is a stronger condition than separability, as defined in Section 3.2, and a weaker condition than additivity, as defined in Section 3.3. In

\footnotetext{
${ }^{5}$ The only time this is with some loss of generality is when there is a cycle containing only separable edges. By assumption, $\succeq$ is a linear order over $X$ and must be acyclic, so this is not possible.
} 
contrast, [1] and [7] make the same separability assumption as used in this paper, but [1] allows for non-monotone privacy preferences and [7] uses a relaxed version of monotonicity.

Perhaps the model closest to ours is [3], which also considers privacy-aware agents with preferences over outcome-privacy pairs. However, the technical quantification of privacy is different in the two models, as [3] considers multiple agents engaging in a single interaction instead of multiple choices by a single agent as in the current paper. In addition, the nature of the results in [3] are different from ours: it studies implementation from a mechanism design perspective, while we study the testable implications of privacy-aware preferences through the lens of revealed preference analysis.

\section{Concluding remarks}

We conclude by describing what our results mean for Alice's story, and for future research on privacy.

Alice makes choices knowing that she is being observed. She thinks that the observer uses revealed preference theory to infer her preferences. She might think that the observer is not sophisticated, and uses revealed preferences naively to infer her preferences over objects. Alternatively, she might think that the observer is sophisticated, and knows that she has preferences for privacy; in this case, the observer tries to infer (again using revealed preferences) Alice's preferences for objects and privacy.

The story of Alice, however, is more "The Matrix" than "in Wonderland." Alice believes that she is one step ahead of the observer, and makes choices taking into account what he learns about her from her choices. In reality, however, the observer is us: the readers and writers of this paper.

$W e$ are trying to understand Alice's behavior, and to infer what her preferences over objects might be. The main result of our work is that such a task is hopeless. Any behavior by Alice is consistent with any preferences over objects one might conjecture that she has (and this is true for any degree of sophistication that Alice may have in her model of what the observer infers from her). Other observers on the internet, such as Google or the NSA, would have to reach the same conclusion.

One way out is to impose additional structure on Alice's preferences. The main result uses separability and monotonicity, which are strong assumptions in many other environments, but that is not enough. We have suggested additive privacy preferences (Section 3.3) as a potentially useful model to follow. Additive preferences do impose observable restrictions on choice, and its parameters could be learned or estimated from choice data. Privacy researchers looking to model a utility for privacy should consider the additive model as a promising candidate.

\section{References}

[1] Yiling Chen, Stephen Chong, Ian A. Kash, Tal Moran, and Salil Vadhan. Truthful mechanisms for agents that value privacy. In Proceedings of the 14 th ACM Conference on Electronic Commerce, EC '13, pages 215-232, 2013. 
[2] Arpita Ghosh and Aaron Roth. Selling privacy at auction. In Proceedings of the 12th ACM Conference on Electronic Commerce, EC '11, pages 199-208, 2011.

[3] Ronen Gradwohl. Privacy in implementation. In CMS-EMS Discussion Paper 1561, 2013.

[4] Ori Heffetz and Katrina Ligett. Privacy and data-based research. Technical report, National Bureau of Economic Research, 2013.

[5] Andreu Mas-Colell, Michael D. Whinston, and Jerry R Green. Microeconomic theory, volume 1. Oxford university press New York, 1995.

[6] Kobbi Nissim, Claudio Orlandi, and Rann Smorodinsky. Privacy-aware mechanism design. In Proceedings of the 13th ACM Conference on Electronic Commerce, EC '12, pages 774-789, 2012.

[7] Kobbi Nissim, Salil Vadhan, and David Xiao. Is privacy compatible with truthfulness? In Proceedings of the 4th Innovations in Theoretical Computer Science, ITCS '14, 2014. To appear.

[8] Lee Rainie, Sara Kiesler, Ruogu Kang, and Mary Madden. Anonymity, privacy and security online. Technical report, Pew Research Center, 2013.

[9] Ariel Rubinstein. Lecture notes in microeconomic theory: the economic agent. Princeton University Press, 2012.

[10] Itamar Simonson and Amos Tversky. Choice in context: tradeoff contrast and extremeness aversion. Journal of marketing research, 1992.

[11] Hal R. Varian. The nonparametric approach to demand analysis. Econometrica, 50(4):945-974, Jul 1982.

[12] Hal R. Varian. Revealed preference. Samuelsonian economics and the twenty-first century, pages 99-115, 2006.

[13] David Xiao. Is privacy compatible with truthfulness? In Proceedings of the 4th Innovations in Theoretical Computer Science, ITCS '13, pages 67-86, 2013. 